\title{
The Frequency of Hot Jupiters in the Galaxy
}

\author{
D. D. R. Bayliss ${ }^{1} \&$ P. D. Sackett ${ }^{1}$ \\ 1 Research School of Astronomy and Astrophysics, The Australian National \\ University, Cotter Rd, Weston Creek, ACT 2611, Australia \\ [daniel@mso.anu.edu.au]
}

\begin{abstract}
The frequency of Hot Jupiters around Galactic dwarf stars is determined from the results of the SuperLupus transit survey and realistic Monte Carlo simulations of the survey efficiency. We find that for Hot Jupiters with mean radii of $1.1 \mathrm{R}_{J}$ and periods between 1 and 10 days, the frequency around dwarf stars is just $0.16 \pm_{0.2}^{0.6 \%}$.
\end{abstract}

\section{Introduction}

Strong observational biases in transit surveys have resulted in the vast majority of transiting planets discovered to date being close-in, Jupiter mass planets ("Hot Jupiters"). However in order to determine how common Hot Jupiters really are in the Galaxy, one must disentangle the discovery rates from the survey biases. The true frequency of Hot Jupiters around dwarf stars in the field is an important ingredient in designing a transit survey and calculating a realistic discovery rate. The frequency is also important in understanding the mechanisms by which these planets form and migrate.

\section{The SuperLupus Survey}

The SuperLupus Survey (Bayliss et al. 2010) is a deep, single field, $0.66 \mathrm{deg}^{2}$ transit survey conducted over three years at Siding Spring Observatory on a 1-m telescope. The survey monitored a field in the constellation Lupus, $11^{\circ}$ off the Galactic plane. The survey was conducted with a five minute cadence, a wide $\mathrm{V}+\mathrm{R}$ filter, and monitored stars in the magnitude range $15<\mathrm{V}<19$. Light curves were produced via Source Extractor (Bertin \& Arnouts 1996) aperture photometry coupled with systematic detrending using SYSREM (Tamuz, Mazeh, \& Zucker 2005). This resulted in a total of 27,000 light curves with $\sigma<0.05$ mag.

Using an automated transit detection algorithm based on the BLS algorithm (Kovács, Zucker, \& Mazeh 2002), ten transit candidates were identified. After spectral typing and radial velocity follow-up, one of these, Lupus-TR-3b, was confirmed as a transiting Hot Jupiter (Weldrake et al. 2008). 


\section{Monte Carlo Simulations}

We used the actual survey window function and photometric uncertainties to create realistic light curves with noise properties similar to that of the survey light curves. We injected transits into the light curves, with transit depths and durations calculated using the statistical properties of the dwarf stars in our field from the Besançon Galaxy Model (Robin et al. 2003). The inserted transits covered a range of planetary radii, periods, phases and inclinations. A total of 800,000 light curves with synthetic transits inserted were generated.

The light curves with inserted transits were passed through the same transit detection algorithm as was used in the actual survey. The recovery fraction as a function of orbital period and planetary radius was then used calculate the efficiency of the SuperLupus Survey. Full details of the Monte Carlo simulations are set out in Bayliss \& Sackett (2010).

\section{Hot Jupiter Frequency}

Using the survey detection efficiency and given just one transiting Hot Jupiter (LupusTR-3b) detected in the field, we estimated the frequency of Hot Jupiters using a Bayesian approach with uniform prior for a period distribution from one to ten days. For a mean planetary radius of $R_{P}=1.1 R_{J}$, we calculate with $90 \%$ confidence intervals that the frequency of galactic dwarf stars in our field with Hot Jupiters $\left(f_{H J}\right)$ is given by:

$$
f_{H J}=0.16 \pm_{0.10}^{0.62} \%
$$

This frequency of Hot Jupiters is slightly lower than that reported from radial velocity surveys: Cumming et al. (2008) report a frequency of $0.65 \pm 0.4 \%$, while Naef et al. (2005) report $0.7 \pm 0.5 \%$. However our calculated frequency is similar to the value reported for other transit surveys where such analysis has been undertaken. The discrepancy between frequencies of Hot Jupiters reported by the radial velocity surveys and transit surveys is most likely due to the different populations monitored by these techniques. Most radial velocity surveys monitor a much brighter sample of stars, which correspond to a population with higher mean mass and metallicity. As such, these populations may have a higher frequency of host Hot Jupiters than the stars monitored by transit surveys.

\section{Discussion}

In the early days of transiting planet surveys, a canonical value of $1 \%$ was used for the frequency of Hot Jupiters around Galactic dwarf stars. It now appears that this was almost an order of magnitude too high, which may have been in part responsible of lower than predicted yields from transit surveys. It also can explain the lack of planets in cluster environments.

With a very high survey efficiency, the Kepler Mission (Basri, Borucki, \& Koch 2005 ) should provide a very robust value for the frequency of Hot Jupiters around galactic dwarf stars. However care will be needed in correctly modeling the effect of the target preselection and detection algorithm. 
Detection and Dynamics of Transiting Exoplanets

\section{References}

Basri, G., Borucki, W. J., \& Koch, D. 2005, New Astronomy Review, 49, 478

Bayliss D. D. R. \& Sackett, P. D. 2010, in prep.

Bayliss D. D. R., Weldrake, D. T. F., Sackett, P. D., Tingley, B. W., \& Lewis, K. M. 2009, AJ, 137, 4368

Bertin, E., \& Arnouts, S. 1996, A\&AS, 117, 393

Cumming, A., Butler, R. P., Marcy, G. W., Vogt, S. S., Wright, J. T., \& Fischer, D. A. 2008, PASP, 120, 531

Kovács, G., Zucker, S., \& Mazeh, T. 2002, A\&A, 391, 369

Naef, D., Mayor, M., Beuzit, J.-L., Perrier, C., Queloz, D., Sivan, J.-P., \& Udry, S. 2005, in ESA Special Publication, Vol. 560, 13th Cambridge Workshop on Cool Stars, Stellar Systems and the Sun, ed. F. Favata, G. A. J. Hussain, \& B. Battrick, 833

Robin, A. C., Reylé, C., Derrière, S., \& Picaud, S. 2003, A\&A, 409, 523

Tamuz, O., Mazeh, T., \& Zucker, S. 2005, MNRAS, 356, 1466

Weldrake, D. T. F., Bayliss, D. D. R., Sackett, P. D., Tingley, B. W., Gillon, M., \& Setiawan, J. 2008, ApJ, 675, L37 